\title{
L'Ufficio perizie FMH compie 20 anni: Rapporto sul periodo 2001
}

\author{
Avvocato Hanspeter Kuhn, segretario generale supplente
}

1 Per facilitare la leggibilità del testo si rinuncia ad indicare, ma resta sottinteso, il genere femminile. Si ringraziano le lettrici per la comprensione.

2 Revisione del Regolamento dell'Ufficio perizie della FMH. Apertura della consultazione. Boll Med Svizzeri 2001; 82(29/30):1585-94 in tedesco; $1602-4$ in francese; pubblicazione del nuovo Regolamento: Regolamento per l'Ufficio perizie della FMH concernente la perizia extragiudiziaria in casi di responsabilità civile del medico. Boll Med Svizzeri 2002;83(6): 267-72.

3 Ott H. Rückblick auf ein Jahr Gutachterstelle für Ärztehaftpflichtfälle. Boll Medici Svizzeri 1983;64(31):1159-60; in francese: Bull Méd Suisses 1983;64(33): 1226-7.

4 Kuhn HP. Aussergerichtliche Gutachterstelle der FMH. 10. Jahresbericht für 1991. Schweiz Ärztezeitung 1992; 73(20):766-7 in tedesco; Bull Méd Suisses 1992;73(22): 852-4 in francese.
Dal 1982 ad oggi, sono state eseguite 2587 perizie FMH per appurare l'esistenza di un errore di diagnosi o di cura. Per la prima volta dall'esistenza dell'Ufficio perizie, quest'anno i periti [1] hanno costatato un errore di cura in più del $50 \%$ dei casi annunciati ad uno dei due Uffici perizie, cioè in 37 casi su 67 (Ufficio perizie di Berna, competente per la Svizzera tedesca e il Ticino).

L'Ufficio perizie FMH non è competente per qualsiasi tipo di contenzioso. Il suo compito consiste nel trasmettere l'incarico di esaminare un caso ad un perito competente, qualora il paziente avesse il sospetto che il medico o l'ospedale sia incorso in un errore di diagnosi o di cura con conseguente danno alla salute e solo nel caso in cui il paziente e l'assicurazione responsabilità civile del medico o dell'ospedale non avessero trovato, nonostante l'impegno, un accordo senza perizia.

Sorprende, che il numero dei casi in cui i periti costatano un errore sia chiaramente diverso tra i due Uffici perizie di Berna e Losanna. Si pone la domanda, se

- nella Svizzera tedesca e nel Ticino gli assicuratori responsabilità civile tendano troppo sovente a negare la presenza di un errore (che poi invece è confermato nella perizia), e

- se nella Svizzera francese, al contrario, i pazienti e i loro avvocati sospettino troppo facilmente la presenza di errori (che poi sono negati dal perito).

Il periodo di revisione del regolamento [2] corrisponde, per un osservatore esterno, con il periodo coperto da questo rapporto. Gli addetti ai lavori sanno che tutto il procedimento peritale è stato riesaminato alfine di ottimizzarne la qualità e i tempi: durata della preparazione e dello svolgimento delle perizie.

\section{Statistica dell'Ufficio perizie per il rapporto 2001}

\section{Metodo}

Da quando esiste l'Ufficio perizie, il compito dei periti è di confermare o negare con il loro esame peritale, l'errore di diagnosi o di cura e di comunicarlo all'Ufficio perizie.
La responsabile dell'Ufficio perizie decide in seguito a quale specialità medica applicare la conclusione. Nei casi in cui la perizia copre più discipline, la scelta cade per quanto riconoscibile, sulla specialità maggiormente coinvolta. Esempio: se il gruppo di periti si occupa in primo luogo d'ortopedia e solo in secondo tempo di anestesia e l'errore è confermato in ortopedia e non in anestesia, la perizia è registrata nella categoria «ortopedia, errore confermato» e non si trova sotto «anestesia, errore negato». La statistica riflette quindi, il risultato per il paziente, il volume del lavoro d'esame svolto dai periti, invece, è registrato solo in parte.

Nelle statistiche mancano le conclusioni dei periti in merito al danno e alla causalità tra errore e danno. Una valutazione statistica di tale questione in base al modello «sì» o «no» lascerebbe un'impressione sbagliata infatti, le conseguenze di un errore confermato possono essere quantitativamente molto diverse. Possono variare da «non si costata nessuna conseguenza negativa» fino «ha causato la morte del paziente».

Sulla lunga durata: in media, negli ultimi vent'anni è stato confermato un errore nel 30\% dei casi, è stato negato nel $67 \%$ dei casi e la questione è rimasta aperta nel $3 \%$ dei casi.

Spaccato cronologico comparativo: nell'agosto del 1983 è stato fatto un rapporto sul periodo da marzo 1982 ad aprile 1983: su 84 casi esaminati, 37 erano conclusi e in sei casi è stato confermato l'errore, in due casi la questione è rimasta aperta e nei restanti 29 l'errore è stato chiaramente negato [3]. Alla fine del 1991, dopo dieci anni d'Ufficio perizie FMH, si arrivava a un totale di 905 perizie. In 212 casi, sempre fino al 1991, l'errore è stato confermato, in 642 casi è stato negato e in 51 casi la questione è rimasta aperta [4].

\section{Perizia comprendente più specialità mediche}

La medicina moderna è diventata più complessa. L'immagine storica del medico che combatte da solo su tutta la linea, per curare i suoi pazienti, non è più attuale nemmeno nell'attività dell'Ufficio perizie. L'anno scorso, circa la metà dei casi esaminati riguardava trattamenti ospedalieri e molti altri casi coinvolgevano una serie di trattamenti svolti da più medici in studio privato. 


\section{Risultati}

Tabella 1

Riassunto delle cifre complessive dal 1982 al 2001.

\begin{tabular}{lccc} 
& Perizie eseguite & $\begin{array}{l}\text { Errore di diagnosi } \\
\text { o cura confermato }\end{array}$ & $\begin{array}{l}\text { Errore di diagnosi } \\
\text { o cura negato }\end{array}$ \\
\hline Losanna e Berna 1982-2000 cura incerto \\
\hline Ufficio Berna 2001 & 2451 & 720 & 1650 \\
\hline Ufficio Losanna 2001 & 67 & 37 & 30 \\
\hline Totale 1982-2001 & 69 & 26 & 43 \\
\hline
\end{tabular}

Tabella 2

Risultati secondo le specialità mediche dal 1982 al 2001.

\begin{tabular}{|c|c|c|c|c|}
\hline Specialità medica & Perizie eseguite & $\begin{array}{l}\text { Errore di diagnosi } \\
\text { o cura confermato }\end{array}$ & $\begin{array}{l}\text { Errore di diagnosi } \\
\text { o cura negato }\end{array}$ & $\begin{array}{l}\text { Errore di diagnosi } \\
\text { o cura incerto }\end{array}$ \\
\hline Anestesiologia & 95 & 26 & 67 & 2 \\
\hline Cardiologia & 9 & 4 & 5 & - \\
\hline Chirurgia della mano & 37 & 11 & 25 & 1 \\
\hline Chirurgia infantile & 13 & 4 & 9 & - \\
\hline Chirurgia generale & 680 & 227 & 429 & 24 \\
\hline Chirurgia del cuore e dei vasi toracici & 16 & 2 & 13 & 1 \\
\hline Chirurgia maxillo-facciale & 17 & 3 & 14 & - \\
\hline Chirurgia ortopedica & 444 & 139 & 294 & 11 \\
\hline Chirurgia plastica e ricostruttiva & 117 & 27 & 88 & 2 \\
\hline Chirurgia viscerale & 4 & 1 & 3 & - \\
\hline Dermatologia & 24 & 8 & 14 & 2 \\
\hline Gastroenterologia & 9 & 1 & 8 & - \\
\hline Ginecologia & 311 & 109 & 196 & 6 \\
\hline Medicina fisica e riabilitazione & 13 & 3 & 9 & 1 \\
\hline Medicina generale & 189 & 63 & 118 & 8 \\
\hline Medicina interna & 167 & 40 & 123 & 4 \\
\hline Medicina d'urgenza & 2 & 1 & 1 & - \\
\hline Nefrologia & 2 & - & 2 & - \\
\hline Neurochirurgia & 55 & 16 & 37 & 2 \\
\hline Neurologia & 18 & 5 & 12 & 1 \\
\hline Oftalmologia & 100 & 25 & 71 & 4 \\
\hline Oncologia & 5 & 3 & 2 & - \\
\hline Otorinolaringoiatria ORL & 96 & 19 & 74 & 3 \\
\hline Patologia & 3 & 2 & 1 & - \\
\hline Pediatria & 47 & 18 & 26 & 3 \\
\hline Pneumologia & 1 & 1 & - & - \\
\hline Psichiatria & 10 & 3 & 7 & - \\
\hline Psichiatria infantile & 1 & - & 1 & - \\
\hline Radiologia & 34 & 11 & 20 & 3 \\
\hline Reumatologia & 8 & 2 & 6 & - \\
\hline Urologia & 60 & 9 & 48 & 3 \\
\hline Totale & 2587 & 783 & 1723 & 81 \\
\hline
\end{tabular}


In concreto, le perizie svolte nel 2001, hanno impegnato i seguenti gruppi di periti:

- Medicina generale - Radiologia (Ufficio perizie Losanna $=$ di seguito $\mathrm{L}$ )

- Medicina generale - Radiologia - Pediatria (Ufficio perizie Berna $=$ di seguito $\mathrm{B}$ )

- Medicina generale - Chirurgia maxillofacciale (B)

- Anestesiologia - Medicina interna (L)

- Anestesiologia - Ginecologia (B)

- Chirurgia - Gastroenterologia Medicina generale - Medicina interna (L)

- Chirurgia - Urologia - Radiologia (L)

- Chirurgia - Ginecologia (B, 2×)

- Chirurgia - Radiologia (B)

- Ginecologia - Radiologia (B)

- Ginecologia - Patologia Medicina generale (B)

- Chirurgia del cuore - Cardiologia Medicina di trasfusione (L)

- Neurochirurgia - Medicina interna (L)

- Medicina interna - Radiologia (B)

- ORL - Dermatologia (L)

- Ortopedia - Anestesia (L, B); assieme a Neurologia (B)

- Ortopedia - Medicina generale (L)

- Ortopedia - Reumatologia (B)

- Pneumologia - Medicina interna (L)

- Psichiatria - Ginecologia (L)

- Radiologia - Ortopedia (B)

\section{Discussione}

Limite della rappresentatività dei casi che approdano all'Ufficio perizie

La statistica dell'Ufficio perizie non è molto rappresentativa per la situazione a livello federale, negli ospedali e negli studi medici, in merito alla responsabilità civile. Per esempio, le 136 perizie svolte l'anno scorso su incarico dei due Uffici perizie $\mathrm{FMH}$, sono da valutare in relazione ai circa 30 fino a 40 casi di responsabilità civile che si verificano in un anno, in un solo, grande ospedale cantonale, non universitario.

L'Ufficio perizie può riferire soltanto sui suoi casi. Non si sa, quanti casi siano sbrigati senza perizia, direttamente dalle assicurazioni, quanti casi siano sottoposti ad esami peritali al di fuori dell'Ufficio perizie FMH e quanti casi arrivino in tribunale.

«Berna»: 37 casi con errore confermato, 30 con errore negato - dipende dal Case-Mix Dal 1996, nell'Ufficio perizie di Berna, il numero dei casi in assoluto è continuamente diminuito. Per contro è aumentata la proporzione dei casi in cui si è confermato l'errore: nel 2000 è arrivata per la prima volta al 50\% e nel 2001, periodo descritto in questo rapporto, questo proporzione d'errori confermata dai periti è stata chiaramente superata. Chi conosce il lavoro dell'Ufficio perizie FMH, non si meraviglia di questi risultati. Da anni l'Ufficio perizie sottolinea che le statistiche annuali dipendono, soprattutto, dal tipo dei casi per i quali è richiesta la perizia.

In merito al ruolo dei consulenti e degli avvocati del paziente: la quota degli errori riconosciuti dipende chiaramente dalla qualità della rete di consulenza medica degli avvocati ai quali si rivolgono i pazienti, fatto che noi abbiamo riconosciuto dal loro modo di lavorare. Nel campo della responsabilità civile in medicina, l'avvocato deve essere innanzitutto traduttore della scienza medica (stessa cosa, per esempio, per un avvocato specializzato in diritto per i brevetti che deve occuparsi di un numero importante di questioni tecniche, per cui ha bisogno di una rete di consulenti). È essenziale procedere ad una valutazione medica della situazione con il paziente, prima di avviare dispendiosi impegni giuridici. Questo significa, in concreto, che in base alla cartella medica e alle radiografie bisogna riflettere, tra paziente o avvocato e il medico consulente, se sia fondato che il paziente perseveri nel suo sospetto, e cioè che sia stato commesso un errore di diagnosi o di cura.

Da quest'analisi medica interna, con componente (auto-)critica, dipende già come poi si scenderà in trattative per trovare un accordo diretto con la compagnia d'assicurazione responsabilità civile dell'ospedale o del medico, senza ricorrere ad una perizia. Ma anche se con l'assicurazione responsabilità civile non si trovasse accordo senza una perizia, la valutazione medica della situazione da parte del paziente è decisiva in merito al se e al come debba essere organizzata una perizia; quanto detto vale indipendentemente dal fatto che per l'esame peritale si faccia capo all'Ufficio perizie FMH, oppure che le parti cerchino e incarichino, direttamente, un perito o un gruppo di periti di loro fiducia. Di conseguenza, consigliamo al paziente di scegliere il proprio avvocato in funzione dei consulenti medici con i quali collabora, e di verificare chi siano gli stessi.

Le questioni propriamente giuridiche, per le quali l'avvocato stesso ha la necessaria competenza specifica, si porranno soltanto dopo che le questioni mediche, in merito all'errore di diagnosi e di cura e le loro conseguenze mediche per lo stato di salute del paziente, sono state chiarite.

In merito al ruolo degli assicuratori di responsabilità civile, degli organismi responsabili degli ospedali, degli assicuratori sociali e del Consiglio federale: la quota, a nostro avviso alta, d'errori ricono- 
sciuti nei casi dell'Ufficio perizie di Berna fa nascere la domanda, se per caso alcuni assicuratori di responsabilità civile abbiano insistito inutilmente per avere una perizia FMH in casi dove una ragionevole valutazione della situazione avrebbe potuto e dovuto portare al disbrigo diretto della faccenda.

Senza un'analisi dettagliata dei fatti, preferibilmente nell'ambito di «closed-claims studies» delle società mediche specialistiche, si può soltanto porre la domanda, ma non si può dare la risposta.

Si impone comunque un'osservazione importante e ovvia: se gli organismi responsabili degli ospedali, cioè cantoni e comuni, le assicurazioni sociali e il Consiglio federale (nella sua funzione d'istanza superiore per litigi concernenti le tariffe) continueranno a sfruttare le risorse disponibili come si spremono i limoni, è prevedibile che aumenti la pressione sugli ospedali affinché scelgano fra le offerte d'assicurazione responsabilità civile i contratti più convenienti. Questa situazione comporta il rischio palese che l'assicuratore chiamato a rispondere, debba valutare molto severamente quale paziente abbia la volontà e i mezzi per chiarire, fino in fondo, il suo sospetto d'errore: cioè superare la fase delle trattative senza perizia, impegnarsi per ottenere una perizia, e nel peggior dei casi, inoltrare istanza giudiziaria. Questo sviluppo non è auspicabile né per i pazienti, né per i medici ospedalieri e nemmeno per il personale curante. Ripetiamo quanto è già stato detto nel rapporto dell'anno scorso: non sono necessari sgravi di prove per il paziente, ma soltanto sufficienti riserve finanziarie presso gli assicuratori responsabilità civile, ciò permetterebbe loro d'indennizzare il danno in modo rapido e corretto.

L'Ufficio perizie di Berna presenta due ulteriori aspetti che colpiscono:

- nei casi in cui, dall'inizio via, era chiaro che bisognava incaricare un gruppo di periti di diverse discipline, l'esame peritale si è concluso con la costatazione d'errore in una misura proporzionalmente superiore alla media, cioè in concreto su 13 dei 15 casi pluridisciplinari;

- nei casi in cui, i preparativi sono stati difficili e notevolmente dispendiosi a causa di dossier incompleti oltre, eventualmente, ad incarichi diffusi e imprecisi, si nota che la perizia raramente si conclude con la conferma dell'errore.

Quest'informazione può essere d'aiuto al paziente coinvolto, all'avvocato e all'assicurazione responsabilità civile che hanno intenzione di chiedere una perizia.
Il paziente presume un errore di diagnosi o di cura, come deve reagire il medico coinvolto?

1. Fissare con il paziente, un appuntamento per discuterne con calma. Chiedere al paziente se desidera farsi accompagnare da una persona di sua scelta.

2. Comunicare rapidamente (!) il caso all'assicurazione responsabilità civile e discutere sul da farsi; chiedere in precedenza l'accordo del paziente (il consenso orale è sufficiente, ma deve essere annotato nella cartella medica). Annunciare il caso all'assicurazione di responsabilità civile, non significa ammettere l'errore!

3. In un ospedale pubblico: svolgere rapidamente un'analisi interna della situazione con il responsabile della divisione ospedaliera e il responsabile della direzione dell'ospedale.

4. Dettare o scrivere al più presto e in modo possibilmente completo, tutto quello che ancora si ricorda sulle fasi decisive della diagnosi e della cura, sotto forma di protocollo («rivedere il film ancora una volta»).

5. Su richiesta del paziente consegnare, senza indugio e gratuitamente, una fotocopia della cartella medica. Le radiografie devono essere messe a disposizione del paziente dietro ricevuta.

6. Quando il colloquio tra medico e paziente non ha portato al chiarimento desiderato, indicare al paziente altre possibilità per verificare il suo punto di vista.

7. Non indirizzare il paziente all'Ufficio perizie senza avere, in precedenza, discusso il caso al telefono, evitando di fare nomi, con la responsabile del competente Ufficio perizie.

8. Ha bisogno di un avvocato il medico coinvolto? L'assicurazione responsabilità civile non ha solo il compito di pagare quando un medico è responsabile di un danno, ma ha pure il dovere di evitare pretese di responsabilità civile ingiustificate. Pertanto il medico non ha bisogno, in fondo, di incaricare un suo avvocato. L'esperienza però insegna, che in certi casi, può essere utile discutere la situazione, in modo informale, con un avvocato indipendente. Una tale analisi della situazione permette inoltre di liberarsi da paure e insicurezze. Di regola, l'assicurazione responsabilità civile non copre queste spese. 
«Losanna»: maggior numero di perizie, due terzi delle quali concludono negando l'errore Nella Svizzera francese risaltano due aspetti. Primo, il numero delle perizie eseguite è alto, anche l'anno scorso è stato proporzionalmente superiore a livello nazionale, infatti, supera in assoluto quello del resto della Svizzera. Il secondo aspetto è che, da anni, il numero degli errori confermati non supera un terzo dei casi sottoposti a perizia. Ciò significa che il paziente, gli avvocati ed eventualmente i loro consulenti medici, nel giudicare la situazione prima di chiedere la perizia, sbagliano due volte di più degli assicuratori responsabilità civile.

A nostro avviso, è legittimo il sospetto che i pazienti (o i loro avvocati), prima di presentare la richiesta di perizia all'Ufficio perizie, non abbiano sufficientemente chiarito la situazione medica o addirittura non l'abbiano chiarita per niente. È vero che le tasse amministrative per la perizia, per motivi sociali e politici, sono fissate ad un livello tanto basso che non copre nemmeno lontanamente le spese vive del lavoro di preparazione sostenuto dall'Ufficio perizie e d'esame dei casi svolto dal perito. Bisogna però tener conto del fatto che la maggior parte dei pazienti incarica un avvocato con pratica privata di presentare la richiesta. Spesso l'onorario degli avvocati già per la sola richiesta e ancor più per la fase della perizia è di diverse migliaia di franchi. Considerato l'importante dispendio di risorse umane e finanziarie che una perizia comporta per tutti i coinvolti (paziente, avvocato, assicuratore, Ufficio perizie, delegati della società medica specialistica e in particolare il perito) sarebbe il caso di esaminare seriamente, se non siano possibili in questo caso, dei cambiamenti concreti:

- in primo luogo, i pazienti e rispettivamente i loro avvocati, prima di generare ingenti spese d'avvocato e dispendio di perizie, dovrebbero curare come regola di partenza, l'organizzazione di migliori consulenze mediche;

- inoltre, le società mediche cantonali potrebbero prendere in considerazione la possibilità di organizzare, a livello cantonale o per tutta una regione, una rete di medici ai quali far capo per un secondo parere. Questi medici sarebbero le persone di contatto a disposizione del mediatore della società medica cantonale, dei mediatori pubblici, delle commissioni di ricorso, delle organizzazioni dei pazienti e delle assicurazioni, e degli avvocati dei pazienti. Il compito di questi medici non sarebbe quello di redigere una perizia, ma quello di dire in base alla cartella medica procurata e messa a disposizione dal paziente, se le attese di successo del paziente fossero sproporzionate, oppure se e cosa potrebbe essere andato storto nella diagnosi e nella cura. Il contributo più importante della società medica cantonale non consisterebbe tanto nel finanziamento di quest'attività, ma nella designazione di medici disposti a svolgere un simile compito e competenti. L'associazione di categoria collaborerebbe così in modo efficace e tempestivo a contenere le spese, ad evitare perizie o addirittura processi inutili.

\section{Sospetto d'errore dei medici che assumono} successivamente la cura del paziente

Molti pazienti hanno preso in considerazione per la prima volta, su indicazione del medico curante successivo, la possibilità di sottoporre a perizia il precedente trattamento. La perizia conferma spesso, in seguito, che il suggerimento era giustificato.

Peraltro si nota che una clinica della Svizzera tedesca, in cui si svolgono interventi chirurgici e un primario di un'altra clinica, pure svizzera tedesca, nonché pochi altri medici praticanti in diverse regioni della Confederazione, inviano molto sovente i loro pazienti dall'avvocato e all'Ufficio perizie e per finire, frequentemente, i periti non costatano nessun errore. Queste «incitazioni a richiedere una perizia, date alla leggera» causano in ogni caso, dai 3000 ai 4000 franchi di spese d'avvocato inutili per il paziente, e il perito o il gruppo di periti sprecano dalle venti alle quaranta ore di lavoro.

A queste persone che tanto facilmente propongono l'esame peritale per chiarire la responsabilità civile, facciamo la proposta di sollecitare parimenti i loro pazienti a riferire in seguito il risultato dell'azione. Così avrebbero modo di verificare la proporzione di successo dei loro consigli ed eventualmente di tirarne le conseguenze dovute. Chiaro è che quando, nella clinica dove subisce l'operazione successiva, il paziente si sente dire che loro devono continuamente correggere gli errori degli altri ospedali e/o medici, anche l'avvocato più sperimentato e diligente non potrà più dissuadere il suo cliente dall'esigere una perizia, a suo parere non indicata, perché rischia di sentirsi chiedere se crede proprio di saperne di più degli specialisti della clinica dove è stata svolta l'operazione successiva.

\section{Lavoro per il nuovo Regolamento, introduzione di un Consiglio scientifico}

Nell'estate 2001, il progetto di revisione del Regolamento è stato inviato alle organizzazioni dei pazienti, agli avvocati, agli assicuratori, ecc. per la consultazione. Parallelamente, è stata esa- 
5 Regolamento per l'Ufficio perizie della FMH concernente la perizia extragiudiziaria in casi di responsabilità civile del medico. Boll Med Svizzeri 2002;83(6):267-72. La documentazione sull'Ufficio perizie con molte indicazioni supplementari si trova sul sito $\mathrm{FMH}$, al seguente indirizzo: www.fmh.ch; Ufficio perizie.

6 Indirizzo per la Svizzera romanda: signora Brigitte Mottet, responsabile dell'Ufficio perizie extragiudiziarie della FMH, casella postale 64, 1010 Losanna, tel. 0216521674 , fax 021 6523385 . minata in modo critico, tutta la procedura interna. In agosto, il Comitato centrale della FMH ha designato un Consiglio scientifico che subito ha potuto prendere posizione sul risultato della consultazione, ancora prima che la proposta definitiva di Regolamento e lo schema della riorganizzata procedura interna, fossero sottoposte, nel novembre 2001, al Comitato centrale.

Il Regolamento riveduto è stato messo in vigore nel febbraio 2002 con la pubblicazione nel BMS 4/2002 [5].

Con le disposizioni rivedute si spera in futuro, di avere una situazione di partenza più chiara, migliorando così sia la qualità, sia la rapidità della procedura peritale. Le prime esperienze, fuori dal periodo considerato in questo rapporto, sono positive.

\section{Ringraziamenti}

Ringrazio per l'impegnativo lavoro svolto nell'interesse di tutte le parti coinvolte, i periti, in modo particolare i medici delegati delle società mediche specialistiche e le due responsabili dell'Ufficio perizie di Berna e di Losanna.

Riconoscere che dappertutto dove si lavora, si possono commettere errori è una costatazione banale. Convivere con questa realtà, per le parti direttamente coinvolte, non è per niente banale. Un sincero ringraziamento va quindi anche a tutti i medici e a tutti gli ospedali che collaborano, in modo aperto e corretto, alle perizie richieste dai pazienti.

\section{Colloquio telefonico preliminare, indirizzo,} documentazione

L'Ufficio perizie FMH, da anni, offre ai pazienti, ai loro avvocati e alle altre persone che consigliano i pazienti, la possibilità di discutere il caso per telefono con la responsabile dell'Ufficio perizie, prima di presentare la richiesta di perizia definitiva. Dove e presso quale persona, in base ai primi accertamenti, sembra plausibile supporre un errore? A quali altre potenziali fonti d'errore si dovrebbe ancora pensare? In cosa potrebbe consistere il danno alla salute? Su quali particolari aspetti, l'Ufficio perizie deve rendere attenti i delegati delle società mediche specialistiche incaricati di proporre il perito? Ecc. Per questi colloqui preliminari bisogna investire mezz'ora o magari un'ora, però, in questo modo si guadagna tempo prezioso nella procedura peritale e si possono evitare in seguito ritardi dovuti a continue, ulteriori domande.

La Svizzera tedesca e il Ticino [6] ottengono la documentazione per presentare la richiesta di perizia, all'indirizzo seguente: Signora Susanne Friedli, Responsabile dell'Ufficio perizie FMH, Casella postale 293, 3000 Berna 16, tel. 031 31208 77, fax 0313119981.

La documentazione completa è attualmente disponibile sul sito FMH al seguente indirizzo: www.fmh.ch; Ufficio perizie. (Attenzione: si spera che il fatto di mettere a disposizione la documentazione sul sito Web, non si riveli controproducente, nella misura in cui si tralascia di discutere per telefono il caso con la responsabile dell'Ufficio perizie, questo colloquio è importante e se fosse trascurato, dovremo riprendere il sistema d'invio della documentazione esclusivamente dietro richiesta scritta.) 